\title{
Martial Arts: Time Needed for Training
}

\author{
David T. Burke* ${ }^{1}$, MD, MA; Marina Protopapas ${ }^{2}$, DO., Paolo Bonato ${ }^{3}$, PhD; \\ John T. Burke ${ }^{1}$, Rpbert F. Landrum ${ }^{1}$
}

Authors' Affiliation:

1. Department of Physical Medicine \& Rehabilitation, Emory University, School of Medicine, Atlanta, Georgia, USA

2. Capitol Spine \& Pain Centers, Herndon, USA

3. Department of Physical Medicine and Rehabilitation, Spaulding Rehabilitation, Boston, USA

\footnotetext{
* Corresponding Author;

Address: Department of Physical Medicine \& Rehabilitation, Emory University, School of Medicine, 1441 Clifton Road NE, Atlanta, Georgia 30322, USA
}

E-mail: dburke2@emory.edu

Received: Aug 24, 2010

Accepted: Dec 28, 2010

Key Words: Martial Arts; Learning Curve; Self-defense; Training; Karate; Tae kwon Do

\begin{abstract}
Purpose: To measure the time needed to teach a series of martial arts techniques to proficiency.

Methods: Fifteen volunteer subjects without any prior martial arts or self-defense experience were recruited. A panel of martial arts experts selected 21 different techniques including defensive stances, arm blocks, elbow strikes, palm strikes, thumbs to eyes, instep kicks and a carotid neck restraint. The critical elements of each technique were identified by the panel and incorporated into a teaching protocol, and then into a scoring system. Two black belt martial arts instructors directed a total of forty-five 45-minute training sessions. Videotaped proficiency testing was performed weekly. The videotapes were reviewed by the investigators to determine the proficiency levels of each subject for each technique.

Results: The techniques were rated by the average number of training sessions needed for an individual to develop proficiency in that technique. The mean number of sessions necessary to train individuals to proficiency ranged from 27 to 38.3. Using this system, the most difficult techniques seemed to be elbow strikes to the rear, striking with thumbs to the eyes and arm blocking.

Conclusions: In this study 29 hours of training was necessary to train novice students to be proficient in 21 offensive and defensive martial arts techniques. To our knowledge, this is the first study that attempts to measure the learning curves involved when teaching martial arts techniques.
\end{abstract}

Asian Journal of Sports Medicine, Volume 2 (Number 1), March 2011, Pages: 31-36

\section{INTRODUCTION}

$\mathrm{T}$ he study of personal self-defense in the United States has gained increasing attention over the past several years. A large number of self-defense and martial arts training programs now exist, with the numbers of these growing in the past decades. Reasons for the increase in demand include self-defense for women as a means of assault/robbery ${ }^{[1]}$ as well as for protection against sexual assault ${ }^{[2]}$. Others have advocated self-defense courses as a means of obtaining a level of physical fitness ${ }^{[3-12]}$. This has also been 
discussed for children, the disabled and the elderly. In a study of motivations for participation in a martial art course, self-defense and physical fitness were the most frequently mentioned. Other more subtle existential and psychological issues have also been mentioned ${ }^{[13]}$. Whatever the reason, a large number of martial arts schools, books and self-defense courses now exist. With various recommendations about the training offered, and the length of training required to graduate to a reasonable level of proficiency, the selection among the available options is often difficult. There seems to be no universally accepted scale by which aerobic training, strength training or level of selfdefense might be expected from any one of the various martial arts or self-defense courses now available to the public.

The suggested time for training to proficiency in various martial arts based techniques ranges from 2 weeks to several years depending on the course and the level of proficiency desired ${ }^{[14]}$. There are no studies, however, which have looked at the number of sessions necessary to sufficiently train individuals in certain techniques. This study sought to assess the number of sessions necessary for individuals to learn a series of martial arts techniques, with the techniques rated on the average number of sessions necessary to gain proficiency.

\section{METHODS AND SUBJECTS}

This study was completed in the fall of 2002. The study was approved by the Institutional Review Board at Spaulding Rehabilitation Hospital, Boston, Massachusetts. All subjects signed informed consents to be included in this study. Subjects were recruited from hospital employees through printed fliers. The flyer requested participation by employees interested in learning self-defense techniques. Individuals who had no prior martial arts or self-defense training and had no medical condition which precluded their ability to participate in an activity involving mild to moderate exertion over a 45-minute period were eligible. There was no age restriction.
The series of martial arts techniques used in this study were identified by a group of four martial arts trained self-defense experts, all of whom had conducted training in self-defense and hand to hand combat techniques. The backgrounds of the experts included Aikido, Tae Kwon Do, Shotokan and military hand-to-hand combat training. The panel of experts was charged with determining a simple but effective series of defensive techniques that would allow for defense against an assailant with or without a knife in an enclosed space. The selected techniques included: defensive stances, turning to face an opponent in that stance, arm blocks of punches and of kicks, block of a knife attack, elbow strikes, palm heel strikes to the chin, thorax or groin, thumb attack to the eyes, kick to the knee, knee strike to the thigh and to the groin and a carotid choke "sleeper" hold (Table 1).

Training began with five minutes of moderate level warm up and stretching. One of the black belt level instructors taught the entire class while a second black belt level instructor moved about the room assisting those who had questions or whose technique needed modification. The techniques were presented, beginning with static stances, and then moving and turning in the stances. The students were then taught the blocking techniques for punches, kicks and knife thrusts. They were then taught the striking techniques.

The arm blocks were taught with the starting position of the hands in an open non-threatening position. When faced with a hook or a straight jab type punch, the subject countered with a forearm block using the radial edge of the forearm as the blocking surface. The participants were taught to use an " $x$ block" with the wrists crossed so overhead to block an attack to the top of the head or low to capture a kick to the groin.

The hand strike technique for longer distances was with the palm of the hand targeting the nose, the xiphoid process and the groin. The strike was taught to occur with the arm in nearly full extension. For a shorter distance strike with the upper extremities, the participants were taught an elbow strike aimed at the mandible when facing the attacker, and to the nose when attacked from behind. In addition, the subjects were taught a thumb strike to the eyes.

The blocking techniques were taught with padded 
Table 1: List of Self-Defense Techniques

Ready Stance
Defensive Stance
Defensive Walking
Defensive Turn
Stalking Stance
Passing
Carotid “sleeper" hold
Blocks: Left/Right/Up/Down/Forward
Weapon Retrieval
Upper Extremity Strike:
a. Elbow Strike to Face
b. Thumb Strike to Eyes/Fingers to Eyes
c. Palm heel strike to the face, xiphoid process and groin
Lower Extremity Strike:
a. Knee to thigh
b. Knee to groin
c. Instep Kick

forearm protectors. When, in the role of the assailant, the participants were asked to strike slowly at first. Increasing intensity was allowed by the instructors, but always aiming just short of the target.

Throughout the training, when testing for proficiency of the blocking techniques, one of the instructors would serve as the assailant in attempt to introduce increasingly realistic assaults.

For kicks the subjects were asked to strike one of two target dummies. For elbow and hand strikes a realistic plastic dummy (Body Opponent Bag tm) was used, with target areas outlined on the face, chest and groin. For kicks, a softer Proman dummy was used, again with the target area outlined.

The salient features of each technique were translated into a rating system. This rating system was then used to organize instructions to emphasize the important points of each technique. The practice dummies were used to assist participants and instructors for gauging the efficacy of targeting.

The carotid restraint was taught using the body opponent bag during the training. For testing the participant was filmed while using this hold on one of the instructors.
All subjects were tested for proficiency on a weekly basis while being videotaped. The tapes were reviewed and scored by a black belt level martial artist blinded to the date on which the video was recorded. The techniques of all subjects were scored for each session. The score for each technique was made, with a notation of whether this score had reached a predetermined level of proficiency for this technique. The number of hours of training was recorded as the first time the technique was judged to have been performed with proficiency. The number of hours of training needed for each individual to reach this proficiency for each of the techniques was then averaged across all participants. This was judged to be the average number of hours needed to train the group to proficiency.

\section{RESULTS}

The study included 14 females and one male ages 2750. Proficiency testing was performed over the twomonth training period, beginning at week two. In Table 
Table 2: Average number of sessions offered before developing proficiency

\begin{tabular}{lc}
\hline Self-Defense Technique & Number of Sessions \\
\hline Rear Elbow Strike to Face & 38.3 \\
Block Down & 37.5 \\
Knife Retrieval & 37.5 \\
Carotid Neck Restraint & 36.1 \\
Thumbs to Eyes & 35.8 \\
Knee Kick to Pelvis & 35.7 \\
Defensive Turn & 35.7 \\
Block Right & 35.7 \\
Block Left & 35.6 \\
Palm Strike to Pelvis & 35.6 \\
Block Up & 34.9 \\
Rear Elbow Strike to Thorax & 34.0 \\
Forward Elbow Strike & 33.6 \\
Palm Strike to Face & 33.3 \\
Palm Strike to Thorax & 32.9 \\
Release from Seated Passenger & 32.8 \\
Instep Kick to Knee & 31.6 \\
Defensive Stance & 29.6 \\
Defensive Walk & 28.6 \\
Ready Stance & 27.0 \\
\hline
\end{tabular}

2, the techniques are listed in order of the average number of hours needed to reach proficiency. The average number of sessions necessary to train the individuals in these techniques ranged from 27 sessions to 38.3 sessions (Table 2). On the last day of training no person was proficient at all techniques. It was also true that there was no technique for which the entire group demonstrated proficiency on the last day. The most difficult techniques (the technique which took the longest for the group to reach proficiency) included the elbow strikes to the rear, striking with thumbs to the eyes and arm blocking with the outer elbow.

\section{DISCUSSION}

The evolution of the techniques involved in the martial arts does not seem to be derived from science, but instead from cultural folklore ${ }^{[15]}$. Most forms of martial arts are thought to have different strengths and weaknesses in terms of offensive and defensive applicability. Currently, the literature lacks a study, which attempts to provide a good assessment of the efficacy of each. As a preliminary step in this evaluation process, this study sought to measure the learning curves involved when teaching a series of martial arts based self-defense techniques.

There were two distinct training issues that seemed relevant to the overall ability of the subjects to achieve and maintain the maximum proficiency. First, there were some techniques that were mastered earlier in the training sessions. Once mastered, the instructors focused on the remaining techniques. Those first learned well were therefore not repeated frequently in subsequent training sessions. These included the "ready stance" and the "release from the seated assailant". To our surprise, the final testing failure rates for these techniques were $60 \%$ and $50 \%$ respectively. It is clear that the presumption that once proficiency had been attained it would be retained throughout the course was 
not correct. We therefore concluded that maintaining proficiency in even the simpler techniques would require continued review and practice so that competency in each skill would not be lost. This issue needs further clarification to be properly addressed for those who need to maintain their skills in all techniques. The rate of such degradation and the need to reinforce would likely vary by technique. Although the prevention of degradation in learning a technique was not addressed in this study, it is certainly relevant for those who wish to learn and wish to teach such techniques.

The second challenge identified was the difficulty that some subjects seemed to have in mastering several techniques. In particular was the inability of many to achieve proficiency in the "back elbow strikes" directed to both the xiphoid process and to the face. The score sheets of these revealed that failure was most often in accurately striking the target. The error for the group associated with a strike to the face was the tendency to direct the blow towards the temple region rather than to the bridge of the nose. The strikes to the xiphoid process most often failed by failing to strike at the target midline and instead strike at the rib area.

Another difficult to train technique was "thumb to the eye". The greatest cause of failure for this technique was the lack of accurate targeting. Although "proficiency" was defined as the ability to strike only one of the two eyes during testing, the failure rate was still high.

Finally, a subjective assessment of power was among the criteria for scoring each technique. Here, the difficulties are layered. First, we did not have the ability to measure the power of each technique and could only estimate the power by viewing the tapes. Second, even if the power of the techniques was quantified in this study, it could not be compared with available data. The reason is that the literature lacks data concerning the critical forces needed to sufficiently disrupt tissue at any of our striking areas. It is likely that this power would differ by target. For instance, it seems reasonable that much less power would be needed to disrupt the tissue of the eye compared with breaking a leg. Besides, it remained unanswered that how great a tissue disruption would guarantee dissuasion of an assailant. Therefore, the inclusion of power in our estimate of proficiency is quite subjective. Further studies are currently planned to address these central issues.

A last issue clearly relevant to the topic of efficacy is that of situational proficiency. It is known among cognitive psychologists that the introduction of a moderate amount of stress to the environment might serve to enhance the performance of that task. If the task is not as well known or the stress is too great, the introduction of stress might cause the task to be performed with less proficiency ${ }^{[16]}$. As the study of self-defense is done under controlled conditions, one could anticipate that there might be subjects, judged to be proficient, who would not perform with proficiency under stress. As the stressors at any given time might vary, we expect one's ability to react to that stressor to vary over time. These are difficult issues that must be entered the equation. These issues fell outside the scope of this study.

\section{CONCLUSION}

This study is the first study of which we are aware that makes an attempt to judge the amount of time needed to teach martial arts techniques to proficiency. The above data reflect the learning curves by which martial arts neophytes acquire this proficiency. These data suggest that approximately 38 hours of training are needed to teach to proficiency the identified defensive and offensive techniques included in this study.

\section{ACKNOWLEDGMENTS}

We would like to acknowledge the participation of the staff of the Spaulding Rehabilitation Hospital, Boston Massachusetts.

Conflict of interests: The authors have no conflicts to report. 


\section{REFERENCES}

1. Hughes PP, Sherril C, Myers B, et al. Self-defense and martial arts evaluation for college women: preliminary validation of perceptions of dangerous situations scale. Res Q Exerc Sport 2003;74:153-64.

2. Easton AN, Summers J, Tribble J, et al. College women's perceptions regarding resistance to sexual assault. J Am Coll Health 1997;46:127-31.

3. Amako M, Oda T, Masuoka K, et al. Effect of static stretching on prevention of injuries for military recruits. Military Med 2003;168:442-6.

4. Brudnak MA, Dundero D, Van Hecke FM. Are the 'hard' martial arts, such as Korean martial, Tae Kwon Do, of benefit to senior citizens? Med Hypotheses 2002;59:485-91.

5. Chen KM, Snyder M. A research-based use of Tai Chi/movement therapy as a nursing intervention. J Holist Nurs 1999;17: 267-79.

6. Wang JS, Lan C, Wong MK. Tai Chi Chuan training to enhance microcirculatory function in healthy elderly men. Arch Phys Med Rehabil 2001;82:1176-80.

7. Brown DD, Mucci WG, Hetzler RK, Knowlton RG. Cardiovascular and ventilatory responses during formalized T'ai Chi Chuan exercise. Res Q Exerc Sport 1989;60:246-50.

8. Chan KM, Au SK, Choy WY, et al. Beneficial effect of one-year Tai Chi in retardation of bone loss inpostmenopausal women. J Bone Miner Res 2000;15:S444 [Abstract].

9. Channer KS, Barrow D, Barrow R, et al. Changes in haemodynamic parameters following Tai Chi Chuan and aerobic exercise in patients recovering from acute myocardial infarction. Postgrad Med 1996;72:349-51.

10. Cheng, Man-ch'ing: Tai Chi Ch'uan: A simplified method of calisthenics for health and self-defense Berkeley, CA: North Atlantic Books. 1981.

11. Hartman CA, Manos TM, Winter C, et al. Effects of T'ai Chi training on function and quality of life indicators in older adults with osteoarthritis. J Am Geriatr Soc 2000;48:1553-9.

12. Jin P. Efficacy of Tai Chi, brisk walking, meditation, and reading in reducing mental and emotional stress. J Psychosom Res 1992;36:361-70.

13. Twenlow SW, Lerma BH, Twemlow SW. An analysis of students' reasons for studying martial arts. Percept Mot Skills 1996;83:99-103.

14. Muromoto W. What is the "Best Martial Arts" for Self-Defense? The Budo Journal of classical Japanese Martial Arts and Culture web site Available at http://www.furyu.com/onlinearticles/bestself.html.

15. Burke DT, Al-Adawi S, Lee YT, Audette J. Martial Arts as Sport and Therapy. J Sports Med Phys Fitness 2007;47:96-102.

16. Gould D, Udry E. Psychological skills for enhancing performance: arousal regulation Strategies. Med Sci Sp Exer 1994;26: 478-85. 